\title{
EDITORIAL
}

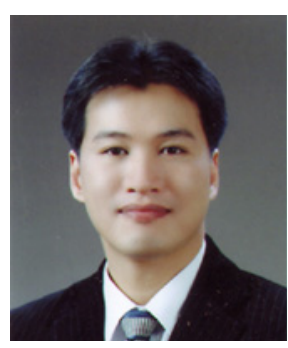

\section{Special Issue on Neural Engineering}

\author{
Chang-Hwan Im \\ (Guest Editor of Special Issue)
}

(C) The Korean Society of Medical \& Biological Engineering and Springer 2012

Neural engineering is an emerging discipline in the field of biomedical engineering that aims to understand, modulate, enhance, or repair neural systems, which are obviously the most complex systems of the human body. During last few decades, neural engineering has been growing rapidly and its applications have been extended from interpreting and processing neural signals to restoring functions of neural systems and interfacing the neural systems with external devices. Despite its short history, neural engineering has now become one of the most important topics in current biomedical engineering research. Considering the above mentioned trends, it seems natural that Biomedical Engineering Letters selected neural engineering as the theme of its first Special Issue. This Special Issue includes five high quality neural engineering related papers, three of which review the current state-of-the-art technologies of this emerging discipline.

The first paper "Inverse Source Imaging Methods in Recovering Distributed Brain Sources" by Ding and Yuan describes the current state-of-the-art technology of electroencephalography (EEG) and magnetoencephalography (MEG) source imaging. This paper reviews principles, recent advancements, and applications of the distributed cortical source imaging techniques, especially focusing on efforts to image human brain activity with high spatial and temporal resolution.

The second paper "Ultrasound as a Noninvasive Neuromodulation Tool" by Jun introduces the ultrasonic neural stimulation technique, which is becoming a promising noninvasive neuromodulation modality. This paper not only reviews recent studies performed to reveal the underlying mechanisms of ultrasonic neuromodulation, but also discusses the future direction of this emerging technology.

Chang-Hwan $\operatorname{Im}(\square)$

Department of Biomedical Engineering, Hanyang University, Seoul, Republic of Korea

Tel : +82-2-2220-2322 / Fax : +82-2-2296-5943

E-mail : ich@hanyang.ac.kr
The third paper "Auditory Brain-Computer Interfaces (BCIs) and Their Practical Applications" by Kim et al. reviews recent studies on auditory brain-computer interface (BCI) paradigms and discusses their prospects in terms of practical applications. Auditory BCI is becoming one of the promising BCI paradigms as it can provide locked-in state patients with impaired visual function with a new pathway for communication. It is expected that this review paper will provide a better understanding of the auditory $\mathrm{BCI}$ technologies, thereby helping biomedical engineers develop a new BCI system.

In the fourth paper "A Dry and Flexible Electrode for Continuous-EEG Monitoring Using Silver Balls Based Polydimethylsiloxane (PDMS)" by Ko et al., a new type of EEG electrodes was proposed. The proposed dry type electrode was manufactured using silver-balls-based PDMS and successfully applied on a comatose patient. It is expected that the proposed dry and flexible electrode will also be used for practical applications such as BCI, continuous EEG monitoring, and neurofeedback.

The last paper "Wrapper Based Wavelet Feature Optimization for EEG Signals" by Garg et al. proposes a new algorithm that can be used for the classification of high dimensional EEG signals. The proposed algorithm named wrapper based wavelet feature optimization (WFO) not only increased the computational efficiency, but also enhanced the predictive power of the system without any loss of relevant information.

In closing this editorial, I would like to express my deepest gratitude to many reviewers, whose professional comments guaranteed the high quality of the selected papers. In addition, I would also like to express my appreciation to Dr. Jae Sung Lee, Managing Editor, and Dr. Sang-Hoon Lee, Editor-in-Chief of the journal Biomedical Engineering Letters, for their help and support throughout the preparation of this Special Issue. I hope you will find this Special Issue helpful for your future study. 